\title{
Encouraging Versatile Thinking in Algebra using the Computer
}

David Tall \& Michael Thomas

Mathematics Education Research Centre University of Warwick Coventry CV4 7AL

Coventry School - Bablake

U.K.

Coundon Rd

Coventry CV1 4AU

U.K.

\begin{abstract}
In this article we formulate and analyse some of the obstacles to understanding the notion of a variable, and the use and meaning of algebraic notation, and report empirical evidence to support the hypothesis that an approach using the computer will be more successful in overcoming these obstacles. The computer approach is formulated within a wider framework of versatile thinking in which global, holistic processing complements local, sequential processing. This is done through a combination of programming in BASIC, physical activities which simulate computer storage and manipulation of variables, and specific software which evaluates expressions in standard mathematical notation. The software is designed to enable the user to explore examples and non-examples of a concept, in this case equivalent and nonequivalent expressions. We call such a piece of software a generic organizer because it offers examples and non-examples which may be seen not just in specific terms, but as typical, or generic, examples of the algebraic processes, assisting the pupil in the difficult task of abstracting the more general concept which they represent.
\end{abstract}

Empirical evidence from several related studies shows that such an approach significantly improves the understanding of higher order concepts in algebra, and that any initial loss in manipulative facility through lack of practice is more than made up at a later stage.

\section{Conceptual difficulties in algebra}

It is well-known, both in everyday teaching experience and in a wide range of empirical studies, that children find great difficulty in understanding algebra (see, for example, Rosnick \& Clement 1980, Matz 1980, Küchemann 1981, Wagner, Rachlin \& Jensen 1984). For a child meeting algebra for the first time there are a number of obstacles which must be confronted and resolved.

First and foremost, there is considerable cognitive conflict between the deeply ingrained implicit understanding of natural language and the symbolism of algebra. In most western civilizations, both algebra and natural language are spoken, written and read sequentially from left to right. There are exceptions 
to this, for instance, numbers in some languages may exhibit a reversal (e.g. 435 is 'vier hundert fünf und dreisig' in German, but 'four hundred and thirty five' in English). However, this is nothing compared with the subtle rules of precedence which occur in algebra. For instance, the expression $3 x+2$ is both read and processed from left to right, however, $2+3 x$ is read from left to right as "two plus three $x$ ", but computed from right to left, with the product of 3 and $x$ calculated before the sum. This difficulty of unravelling the sequence in which the algebra must be processed, conflicting with the sequence of natural language, we term the parsing obstacle. It manifests itself in various ways, for example the child may consider that $a b$ means the same as $a+b$, because they read the symbol $a b$ as $a$ and $b$, and interpret it as $a+b$. Or the child may read the expression $2+3 a$ from left to right as $2+3$ giving 5 , and consider the full expression to be the same as $5 a$.

Prior to the introduction of algebra, children become accustomed to working in mathematical environments where they solve problems by producing a numerical "answer", leading to the expectation that the same will be true for an algebraic expression (Kieran 1981). An arithmetic expression such as $3+2$ is successfully interpreted as an invitation to calculate the answer 5, whereas the algebraic expression $3+2 a$ cannot be calculated until the value of $a$ is known. This unfulfilled and erroneous expectation we term the expected answer obstacle. It causes a related difficulty, which we term the lack of closure obstacle, in which the child experiences discomfort attempting to handle an algebraic expression which represents a process that (s)he cannot carry out.

Another closely related dilemma is the process-product obstacle, caused by the fact that an algebraic expression such as $2+3 a$ represents both the process by which the computation is carried out and also the product of that process. To a child who thinks only in terms of process, the symbols $3(a+b)$ and $3 a+3 b$ (even if they are understood) are quite different, because the first requires the addition of $a$ and $b$ before multiplication of the result by 3, but the second requires each of $a$ and $b$ to be multiplied by 3 and then the results added. Yet such a child is asked to understand that the two expressions are essentially the same, because they always give the same product. Such a child must face the problem of realizing that the symbol $3 a+6$ represents the implied product of any process whereby one takes a number, multiplies it by 3 and then adds 6 to the result. This requires the encapsulation of the process as an object so that one can talk about it without the need to carry out the process with particular values for the variable. When the encapsulation has been performed, two different encapsulated objects must then be coordinated and regarded as the "same" object if they always give the same product - a task of considerable complexity. 
Faced with such obstacles, it is no wonder that so many children fail. To cope with these difficulties, traditional teaching tends to emphasize the calculation and manipulation of algebraic expressions - teaching children the rules of algebra so that they develop the necessary manipulative ability. "Do multiplication before addition", "calculate expressions in brackets first", "collect together like terms", "of means multiply", "add the same thing to both sides", "change sides, change sign", "to divide, turn upside down and multiply", etc etc. It is hoped that once the child is able to carry out the rules consistently, then understanding will follow, but it is a forlorn hope. When algebra is taught as an essentially manipulative activity, following a sequence of mechanistic rules, it is only to be expected that a poor understanding of the subject prevails.

We hypothesise that as soon as children are unable to give meaning to concepts, they hide their difficulties by resorting to routine activities to obtain correct answers and gain approval. Once committed to such a course, it easily degenerates into a never ending downward spiral of instrumental activity: learning the "trick of the week" to survive, soon leading to a collection of disconnected activities that become more and more difficult to coordinate, even at a purely mechanistic level. Therefore the beginning phase of the subject - giving meaning to the variable concept and devising ways of overcoming the cognitive obstacles - is fundamental to laying a foundation for meaningful algebraic thinking.

\section{The continuing need for algebra}

One way to avoid the difficulties so far acknowledged is to reduce the amount of algebra taught, or not to teach it at all. In the UK, the National Curriculum now makes fewer demands on algebra for 16 year olds than was previously the case, and there are moves elsewhere to reduce formal algebra by using more numerical problem-solving (see, for example, Leitzel \& Demana 1988).

We are convinced that the reduction in algebra content - certainly for the average or above average pupil - is a profound error, based on a view of seemingly insurmountable difficulties which have occurred in a pre-computer paradigm. Certainly there is a stage where the introduction of algebra makes matters more difficult (for instance, the question "I am thinking of a number and twice it is six, what is the number?" is more likely to produce a correct response than "solve $2 x=6$ "). But soon there comes a stage in solving slightly more complicated problems where the lack of algebra can bcome a serious handicap. For instance, Gardiner set the following problem in a school mathematics contest:

Find a prime number which is one less than a cube. Find another prime number which is one less than a cube. Explain!

(Gardiner 1988) 
Students soon find two cubed minus one is prime, but looking for patterns in the numbers that turn up by cubing and subtracting one (2 gives 7, 3 gives 26, 4 gives 63, etc) can give interesting red-herrings. (For instance, when the number is odd the result is even, but when the number is even the result is odd and the question needs further investigation.) But applying the process to the number $n$ gives $n^{3}-1$ and anyone with a little experience in algebra can see that this factorizes to $(n-1)\left(n^{2}+n+1\right)$, which is prime only when $n=2 \ldots$ We thus see that there is a stage in the curriculum when the introduction of algebra may make simple things hard, but not teaching algebra will soon render it impossible to make hard things simple.

We believe that, whilst the initial difficulties cannot be totally avoided, they are exaggerated by the teaching of algebra in a context in which the symbolism does not make sense to the vast majority of pupils, and that the success rate can be significantly improved by giving a coherent meaning to the concepts by using a computer. The principles which we will shortly describe apply throughout mathematics and in the next section we formulate a wider theoretical framework before returning to the specific case of algebra.

\section{Versatile Thinking in Mathematics}

To be able to be a successful mathematician requires more than the ability to carry out a succession of mechanistic steps, be they steps in carrying out a numerical calculation, solving a linear equation, differentiating a composite function, or writing down a mathematical proof. What is also required is an overall picture of the task at hand, so that the appropriate solution path can be selected and any errors that occur are more likely to be sensed and corrected. Thus the sequential/logical/analytic way of carrying out a succession of mathematical processes needs to be complemented by a global/holistic overall grasp of the context.

The differences between these forms of thinking has been a focus of thought for several centuries. Descartes (1628), for example, contrasted the intuition of an immediate perception of connections between concepts with chains of logical deduction required to give formal relationships. Poincaré (1903) distinguished between those mathematicians who thought in a predominantly sequential/deductive mode, and those whose work developed more through intuition.

Krutetskii (1976, p. 326) divided his mathematically gifted pupils into analytic, geometric and harmonic types, according to their preferences for verbal-logical, visual-spatial, or a combination of the two. He was of the opinion that the analytic and geometric types mentioned by Poincaré should be acknowledged as somewhat limited because they tend to specialize only in restricted areas of mathematics. Although representatives of such extreme 
types can be successful in school mathematics, they are liable to experience difficulties which are a consequence of their limitations.

In the last two decades there has been considerable speculation that the sequential/analytic and global/holistic modes of thinking may be due to different methods of processing in different parts of the brain. Several physiological studies (e.g. Sperry, Gazzaniga \& Bogen 1969, Seamon 1974, Gazzaniga 1974, Popper \& Eccles 1977) promoted a model of thinking as a unified system consisting of two, qualitatively different processors, linked via the corpus callosum (the connection between the two hemispheres), with the rapid flow of data between them and the processing done by them, controlled by a control unit. In this model one processor - usually in the major, left hemisphere of the brain of right-handed individuals - is a sequential processor involved with logical, linguistic and mathematical activities (see e.g. Bogen 1969). The studies suggested that the control unit appears more likely to be seated in this hemisphere, possibly accounting for its dominance. The other processor, in the minor, right hemisphere, is a fast, parallel processor, able to make global decisions, and being the primary centre of visual and mental image reasoning (Bogen 1969).

Following criticism and further research, Gazzaniga (1985) proposed a modified model in which the brain is organized into many relatively independent functioning units working in parallel, with the control unit mentioned above interpreting and coordinating the product of these various units. He gives examples in which individual functions may lie in the left or right brain of different individuals. According to his theory, what is important is not where the different abilities are located in the brain, but how they operate and interconnect. Some units, related to verbal, aural and linguistic abilities are able, by their very nature, to give conscious verbal output to the individual and constitute a sequential/verbal mode of thought. Others, relating to visual, tactile and other senses can give only gestalt, non-verbal output, relating to what we have characterized as global/holistic thought. This suggests that there may be aspects of visual thinking involved in understanding mathematics which are less easily verbalised and hence less likely to be valued.

The cognitive model proposed by Thomas (1988) stresses the need for cognitive integration of the two qualitatively different modes of thinking, with both being actively promoted in teaching and thus made available in the mind of the mathematics student. Following Brumby (1982), we use the term versatile thinking to refer to the complementary combination of both modes, in which the individual is able to move freely and easily between them, as and when the mathematical situation renders it appropriate.

In general, the global/holistic side of the brain's activity has been conceived as intuitive and visual. Certainly, if its output is holistic, with links between concepts made simultaneously with no logical or sequential relationships being 
apparent, then it qualifies for the term "intuitive". However, we believe that this intuition can be honed to link in with the sequential/logical thinking processes and therefore be of value in giving an overall view of the mathematical structure. We also believe that visual ideas are not only graphical and geometric. Symbolism is viewed through the eye, and whilst it may be processed sequentially through scanning the expression, it may also be taken in holistically, in chunks, and this side of visualization is very important in algebra.

\section{Versatile thinking in algebra}

An illustration of versatile thinking arises from the following type of question: Factorize : $(2 x+1) 2-3 x(2 x+1)$.

Many secondary-school pupils who view factorization as a process seem locked into a serialist/analytic mode where they work from left to right "multiplying out brackets", "collecting together like terms" and factorizing the resulting quadratic function. Apparently unable to break free from such a serialist strategy, few seem able to apply the versatility of thought necessary to switch from an analytical approach to a global/holistic one and "see" that $2 x+1$ is a common factor, hence moving directly to the answer, with a minimum of analytic processing.

One of the reasons for the prominence of a serialist line of thinking in questions like the one above, would seem to be the emphasis in the pupil's mind on obtaining the product or answer, with the process existing as a means to that end, rather than being a conceptual entity to think about in its own right. This implies that a pupil may be able to obtain a factorization or "product" by a sequential/analytic process without fully understanding conceptually what a factorization is.

It should be noted that it is possible for an individual to carry out an algorithmic process without abstracting from it an understanding of the concepts embedded in the process. They may even be able to encapsulate the process as an entity that they can think about without understanding its constituent concepts. This may then be repeated at a later stage when, for example, pupils trying to find the highest common factor of several algebraic expressions, using a strategy such as that mentioned above would need to learn another process rather than being able to deduce the answer from their understanding of their previous processes. Their progress is thus characterised by a sequence of process management tasks leading to instrumental understanding, rather than the construction of true relational understanding in the sense of Skemp (1976). It is our belief that the educator should encourage relational understanding not through enforcement of the process itself, but through versatile thinking that reflects on the construction of vital concepts as well as encapsulation of the process. 


\section{Using the Computer to promote Versatile Thinking}

Versatile thinking requires the availability of cognitive interaction between concepts represented by imagery as well as symbolically and verbally. An environment encouraging the formation and manipulation of such cognitive structures should be more likely to produce versatile thinking.

Skemp 1979 proposes three different modes in which the individual can mentally construct concepts: by interaction with the real world (which he terms "actuality"), by interaction with other people, and by reflection on concepts in the mind and the relationships between them. Tall 1990 divides the first of these modes into two:

- interaction with inanimate objects which are passive so that the user must manipulate them him(her)self

and

- interaction with systems, such as computer software, which are cybernetic, and react to the individual's actions according to pre-programmed and predictable rules,

This gives four modes of interaction for concept formation:

- with passive objects

- with cybernetic systems

- with other persons

- by internal reflection.

Of these four, the cybernetic system offers potentially a more consistent mode of building concepts by providing consistent feedback which may be predicted and tested. For instance, if the statement

$$
\mathrm{A}=3
$$

is typed in BASIC, followed by

PRINT A+2

the computer responds with the number 5 . One may then conjecture what will happen if one types

PRINT A+3

or

$\mathrm{B}=\mathrm{A}+2$

PRINT B

and so on, in order to begin to formulate theories about the consistency with which the language handles the symbols.

Of course, the symbols are treated slightly differently in BASIC from standard algebra, for instance, multiplication must be written explicitly in 
BASIC but may be implicit in algebra. It is therefore helpful to program software in which standard algebraic notation is allowed and is evaluated numerically in the usual way. We therefore designed software called the algebraic maths machine which allowed several individual letters to stand for variable numbers, and accepted up to two expressions which could be evaluated to compare the results of calculations (figure 1).

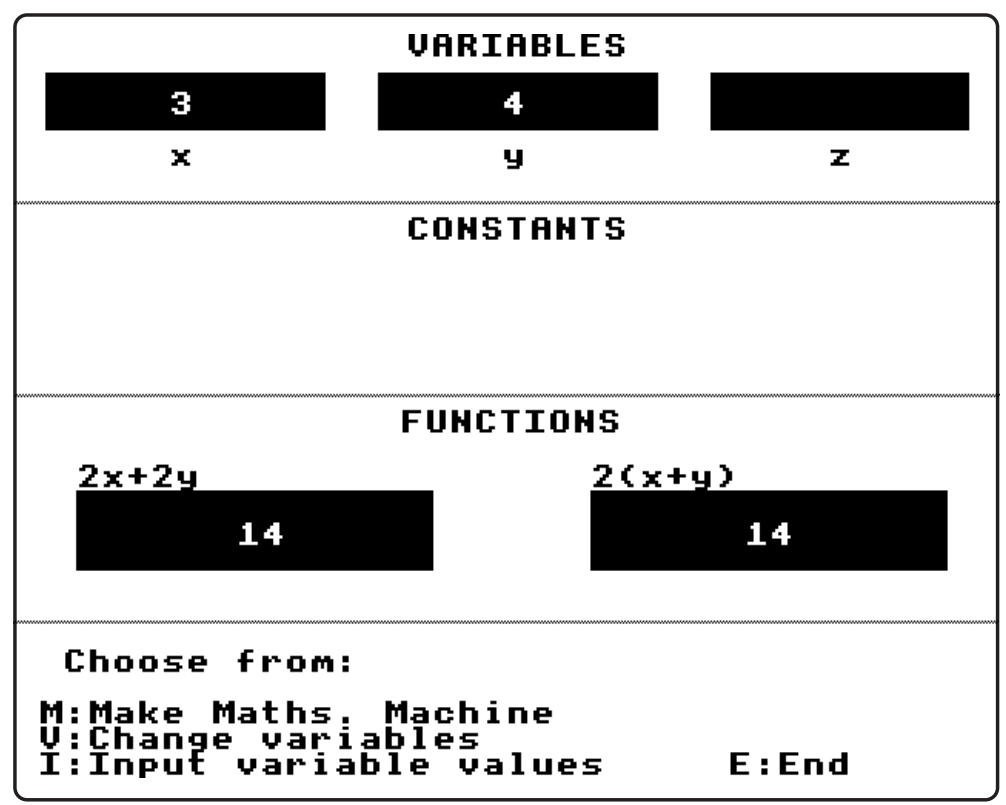

Figure 1

By evaluating expressions such as $2(x+y)$ and $2 x+2 y$ for various numerical values of $x, y$, it is then possible for pupils to experience the fact that different expressions evaluate to the same values every time, and to predict and test what might happen with other expressions, such as $2+3 a$ and $5 a$, or, at a more advanced level, $\left(x^{2}-1\right) /(x-1)$ and $(x+1)$.

Such software, that allows exploration of examples, and non-examples of concepts (in this case equivalent and non-equivalent expressions), is termed a generic organizer (Tall 1986). It encourages the individual to manipulate examples, to predict and test, to develop experiences on which higher-level abstractions may be built.

In general generic organizers may be passive or cybernetic. For instance, Dienes blocks, embodying some of the principles of place value in physical materials, are passive. They require the user to act upon them and reflect on the result of these actions to build up the abstract idea of place value (and to move to the more subtle understanding that each position may be represented symbolically in the same way). The algebraic maths machine is a cybernetic generic organizer, designed to help the pupil abstract the notions of variable and of equivalent algebraic expressions. Again it will require action on the part of teacher and pupil to assist the pupil to abstract the underlying concepts. 
We initially proposed quite subtle theories as to why the algebraic maths machine might promote versatile thinking in which equivalent expressions might be viewed holistically as being the same (Thomas \& Tall 1988). We subsequently suspected the truth to be rather more prosaic. Although the pupil has to type in each expression (in BASIC programming or the algebraic maths machine), and therefore processes them in the normal left to right scansion, the computer carried out all the intervening evaluations. Thus the pupil is relieved of the burden of the evaluation process and simply, by not having to carry it out, is able to focus on the product of the evaluations and to predict and test why different looking expressions may always give the same value.

\section{Activities to promote versatile thinking in algebra}

In addition to the cybernetic organizer, the pupils played with an inanimate organizer, consisting of a cardboard maths machine, which gave them a physical analogy to the process by which BASIC uses letters as variables. This simply consists of two large sheets of card, one representing the computer screen, the other having boxes drawn on it to represent places to store numbers within the computer. In addition there are small pieces of cardboard with letters on them, to place above the stores as labels and numbers to place inside the stores as values

The programming activities in BASIC, such as

$$
\begin{aligned}
& \mathrm{A}=3 \\
& \mathrm{~B}=\mathrm{A}+1 \\
& \text { PRINT B }
\end{aligned}
$$

were carried out analogously by a group of pupils on the cardboard machines. Someone placed a message on the screen to say $\mathrm{A}=3$, and a message was passed to someone else in charge of the stores. (S)he looked to see if there was a store labelled $\mathrm{A}$, and if there was not, a new store was labelled. Then the store labelled $\mathrm{A}$ had the number 3 placed insided. Next the message $\mathrm{B}=\mathrm{A}+1$ was passed to the store-keeper, who made sure that a store was labelled $B$ in the same way, looked into store $\mathrm{A}$ to find the value was 3 , added 1 to get 4 , then placed a card marked 4 in the store $B$. Finally when the PRINT B command was passed over, the store-keeper saw that $B$ contained 4 , and found another card with this number written on and passed it back to be displayed on the carboard screen.

The classroom was flexibly organized. Only two or three computers were needed to service a class of twenty or more pupils, because the others could work on cardboard maths machines.

The setup was intended to involve all four modes of reality construction: 
- interaction with the cardboard maths machine (passive organizer)

- interaction with the algebraic maths machine (cybernetic organizer)

- interaction between the teacher and pupils and between the pupils themselves

- personal reflection on the meaning of the notation.

Initially the curriculum activities were designed:

- to encourage the mental image of a letter as a store label representing a single number which could be changed and, by extension, could hold any of a variety of numbers,

- to consider the evaluation of expressions and equivalent expressions in BASIC notation and in standard algebraic notation.

The pupil could think holistically of a variable as a store labelled with a letter, and of an expression either as a process on the cardboard maths machine or as a product in BASIC or in the algebraic maths machine. By giving meaning to the concept of variable and expression in this way it was hypothesized that the pupils would have a better foundation to construct the meaning of algebraic symbolism.

The parsing obstacle was attacked by discussion between teacher and pupils, then encouraging pupils to reflect on the possible meaning of different expressions, using BASIC and the algebraic maths machine to build and test theories as to the meaning of the notation, the order of evaluation of expressions and the nature of equivalent expressions. We hypothesized that the expected answer obstacle would be less problematic in the computer environment as letters and algebraic expressions had a physical function in the cardboard maths machine and are typed into the computer and seen by the pupil, thus giving a concrete meaning to the symbolism. Given number values for the variables, the expressions could be evaluated, in the same way as arithmetic expressions. Thus the pupils could think of expressions as being potentially evaluable, yet talk about them as conceptual entities, reducing the problem of the lack of closure obstacle. Finally, the process-product obstacle was faced squarely by seeing that the expression represented both process (in the cardboard maths machine) and product (in the algebraic maths machine and in BASIC). As the computer performed the process in BASIC and the Algebraic Maths Machine, this allowed the pupils to concentrate on the notation as product and to think of it as a conceptual entity.

The whole module, designed to last for three weeks, with four one-hour lessons a week, has subsequently been published under the title "Dynamic Algebra" by the Mathematics Association (Tall \& Thomas 1989a). 


\section{The Experiments}

In the first pilot experiment pupils aged 11/12 in the final year of a Middle School were divided into 21 matched pairs. One half of the pairs were given the Dynamic Algebra Module over a three week period whilst the other half did no algebra. It would therefore be expected that this pilot study showed improvements in the understanding of algebra (Thomas \& Tall 1986). What is more significant is that one year later, when all the children had moved schools and studied algebra in a traditional way, a follow-up study indicated that there were still measurable differences in performance on algebra.

All the pupils transferred to Secondary Schools at the end of the school year. Eleven of the matched pairs transferred to the same school and were put into the same mathematics sets, so that during their first year at the school (age $12 / 13$ years) they received an equivalent tuition in algebra. At the end of that year they were again given the algebra test used in the original study to see what the longer term effects might have been. A summary of the results and a comparison with their previous results - using the $t$-test for non-independent samples - are given in table I. We see that, over one year after their work on the basic concepts of algebra in a computer environment, they were still performing significantly better than those who had not experienced such work. (The relatively low scores - approximately half marks out of a maximum of 71 - occur because conceptually harder questions from the CSMS tests were included on the test. The performance of the control students was typical of a large sample tested by the CSMS project.)

\begin{tabular}{|l|c|c|c|c|c|c|c|c||}
\cline { 2 - 10 } \multicolumn{1}{c|}{} & $\begin{array}{c}\text { Exper. } \\
\text { mean } \\
(\max =71)\end{array}$ & $\begin{array}{c}\text { Control } \\
\text { mean } \\
(\mathrm{max}=71)\end{array}$ & $\begin{array}{c}\text { Mean } \\
\text { diff. }\end{array}$ & S.D. & $N$ & $t$ & $d f$ & $p$ \\
\hline Post -test & 32.55 & 19.98 & 12.57 & 10.61 & 21 & 5.30 & 20 & $<0.0005$ \\
\hline Delayed post-test & 34.70 & 25.73 & 8.47 & 11.81 & 20 & 3.13 & 19 & $<0.005$ \\
\hline One year + later & 44.10 & 37.40 & 6.70 & 7.76 & 10 & 2.59 & 9 & $<0.025$ \\
\hline
\end{tabular}

Table I : performance of 11 matched pairs on simple algebraic problems

We may conclude then that there are significant longer term benefits to be had from the introduction of a module such as the Dynamic Algebra package, with its emphasis on conceptualisation and use of mental images rather than on skill acquisition, prior to the more formal learning of algebra.

In order to extend this comparison between the two approaches mentioned above a larger scale experiment was arranged whereby a direct comparison might be made between the Dynamic Algebra Module and a traditional skill based module. The subjects of this second experiment were taken from 6 mixed ability forms in the first year of a mixed ability 12-plus entry comprehensive school (i.e. 12/13 year olds). On the basis of an algebra pre- 
test based on the CSMS algebra test, and correlated with it, it was found to be possible to organise 57 matched pairs from the classes.

In the first stage of the comparison the experimental group received the Dynamic Algebra Module during their normal mathematics periods over about 4 weeks (about 12 hours). During this time the control group were taught elementary algebra using a traditional skill based module of work used in the school over some years, and covering basic simplification and equation solving in one unknown. Since this point in the experiment gave an ideal opportunity to compare the effects of each treatment thus far after each group had finished its work they were given the same test again and a close comparison of their performance made.

Six months later, all the pupils were given the same traditional module for a two week period, the controls as revision, the others for the first time, followed by a repeat of the test. After the post-test of this experiment a crosssection of 18 pupils, 11 experimental and 7 control, were given a semistructured interview lasting about twenty minutes. During the interviews, which were recorded, the pupils were required to attempt certain key questions and to explain their thinking and strategies.

\section{The Results}

The overall results, as seen in table II, showed that there was no significant difference in performance between the groups on the algebra test in the post test, but that, after the second post test the difference becomes significant.

\begin{tabular}{|l||c|c|c|c|c|c|c|c||}
\cline { 2 - 9 } \multicolumn{1}{c|}{} & $\begin{array}{c}\text { Exper. } \\
\text { mean } \\
(\mathrm{Nax}=48)\end{array}$ & $\begin{array}{c}\text { Control } \\
\text { mean } \\
(\mathrm{max}=67)\end{array}$ & $\begin{array}{c}\text { Mean } \\
\text { diff. }\end{array}$ & S.D. & $N$ & $t$ & $d f$ & $p$ \\
\hline Post -test & 36.0 & 35.9 & 0.10 & 10.46 & 48 & 0.06 & 47 & n.s \\
\hline Second post-test & 42.1 & 39.3 & 2.76 & 8.91 & 47 & 2.08 & 46 & $<0.025$ \\
\hline
\end{tabular}

Table II : Performance of 48 matched pairs on algebra post-tests

A different picture emerges on consideration of the results of individual questions. Perhaps, not surprisingly, there are some questions where the control group performed significantly better than the experimental group and these tended to be those questions involving the sort of skills, particularly associated with simplification of expressions, which they had been taught (see table III, which uses the $z$-statistic to test the difference between the means of two normal distributions). However, there were some questions on which the experimental group did noticeably better, and significantly so in some cases. As table IV shows, these were questions which are of a harder conceptual nature and require a higher level of understanding, as defined by Küchemann (1981), including in the case of several of the questions the concept of letter as a generalised number or variable. Thus it appears that there were differential 
effects from the two approaches in respect of algebraic skills on the one hand and deeper conceptual understanding on the other.

\begin{tabular}{||l|c|c|c|c||}
\hline $\begin{array}{c}\text { Question } \\
\text { (asked of 48 matched pairs) }\end{array}$ & $\begin{array}{c}\text { Experimental } \\
\text { proportion } \\
\text { correct }\end{array}$ & $\begin{array}{c}\text { Control } \\
\text { proportion } \\
\text { correct }\end{array}$ & $z$ & $p$ \\
\hline Multiply $3 c$ by 5 & 0.10 & 0.44 & 3.67 & $<0.0005$ \\
\hline Simplify $3 a+4 b+2 a$ & 0.52 & 0.75 & 2.30 & $<0.05$ \\
\hline Simplify $3 b-b+a$ & 0.33 & 0.63 & 2.83 & $<0.005$ \\
\hline Simplify $3 a+4+a$ & 0.42 & 0.54 & 1.21 & - \\
\hline Gjigsaws and $H$ jigsaws $=?$ & 0.58 & 0.81 & 2.45 & $<0.01$ \\
\hline
\end{tabular}

Table III : Algebraic skills

\begin{tabular}{||l|c|c|c|c||}
\hline \multicolumn{1}{|c|}{$\begin{array}{c}\text { Question } \\
\text { (asked of 48 matched pairs) }\end{array}$} & $\begin{array}{c}\text { Experimental } \\
\text { proportion } \\
\text { correct }\end{array}$ & $\begin{array}{c}\text { Control } \\
\text { proportion } \\
\text { correct }\end{array}$ & $z$ & $p$ \\
\hline For what values of $a$ is $a+3>7 ?$ & 0.35 & 0.13 & 2.63 & $<0.005$ \\
\hline For what values of $a$ is $6>a+3$ ? & 0.25 & 0.06 & 2.53 & $<0.01$ \\
\hline $\begin{array}{l}a+b=b-\text { always, never, } \\
\text { sometimes ... when ? }\end{array}$ & 0.33 & 0.15 & 2.15 & $<0.025$ \\
\hline $\begin{array}{l}M+P+N=N+M+R,- \text { always, } \\
\text { never, sometimes when? }\end{array}$ & 0.44 & 0.29 & 1.48 & - \\
\hline Perimeter of rectangle $D$ by 4 & 0.52 & 0.27 & 2.50 & $<0.01$ \\
\hline Perimeter of rectangle 5 by $F$ & 0.52 & 0.29 & 2.29 & $<0.025$ \\
\hline Which is larger: $2 n$ or $n+2$ ? & 0.08 & 0.00 & 2.04 & $<0.025$ \\
\hline
\end{tabular}

Table IV : Conceptual questions

\section{Evidence For Versatility and Conceptual Understanding}

The results of our work suggest differential effects between the computerbased approach to algebra, with its emphasis on letters as generalized numbers and the traditional skill-based type of module with its emphasis on acquiring manipulative skills. It seems that the computer work promoted a deeper conceptual understanding, whilst the other work, as expected, initially facilitated better surface skills. However, when the computer module was combined with the skill-based one then it led to a superior overall performance without detrimental effect on skills.

A significant fact here is that the experimental students are initially performing better at skills which are considered to be of a higher level. In other words, the order of difficulty of concepts in the computer paradigm is different from that in the traditional paradigm. This makes the general point that empirical research in a pre-computer paradigm may no longer be appropriate for a computer paradigm. The relative degree of difficulty of concepts is not absolute, it may be paradigm-dependent.

Given the evidence from the earlier pilot test that the difference in performance persisted over a longer period, we waited for eighteen months and gave the students a repeat of the post-test. On this occasion the difference 
between experimental and control groups was no longer statistically significant (Thomas \& Tall 1989b). During this period the classes had been reorganized in a way which has led to a variety of different experiences for pupils matched in pairs during the experiment. This suggests that, although computer experiences may be able provoke different kinds of understanding in the short and medium term, if these experiences are not continued then their effect may wane in the face of the overwhelming influence of more recent experiences.

However, understanding of algebraic concepts may not be evidenced by test performance alone, since, for example, correct answers may be the result of incorrect understanding. In order to examine the pupils' understanding of algebra beyond their test performance it is useful to refer back to interviews and a questionnaire administered after the second post-test.

\section{The Interviews}

The transcript of the interviews reveals, in the language used by the pupils in each of the groups, the versatility of thought underlying the better performance of the computer group.

The superior relational understanding and versatility of thought of those who had used the Dynamic Algebra module strikingly manifested itself in several different ways, including:

(i) The attempts by the experimental group to explain and offer reasons for their thinking, compared with the control pupils greater concern with surface operations in their comments.

(ii) An improvement in encapsulation of processes, shown by the ability of the computer group to discuss processes without having to carry them out first and to exhibit global knowledge of a question in their comments.

(iii) The ability of the computer group pupils to take a global view of a problem, rather than being pressed into processes implied by the operations present in the notation.

The following examples taken from the transcripts of the interviews show these manifestations of improved versatile thinking:

Question: Solve $2 p-1=5$.

The confusion in thinking which may result from instrumental understanding is illustrated by one of the control pupil's explanations for this question :

Pupil C2: $2 p$ minus 1 equals 5 . If you add the 1 to the 5 that's 6 , so because there's no other minus $p$ I forget the $p$ and do the $2 p$ minus 1 equals. If you add the 1 to the 5 which is 6 and then you take 1 from the 6 . No, I don't get that. I know I've done it but...

Interviewer : What would the value of $p$ be did you say?

Pupil C2 : Six. 
Here the explanation is solely in terms of the operations with no reasons for their use being cited. This may be compared with the following reasoning from one of the experimental group pupils :

Pupil E2 : Well find out what minus $1 \ldots$ so you would add 1 to that so you get rid of the 1 , so that would be 6 and then its obvious that 2 times 3 equals 6 , so $p$ would be 3 .

The pupils in the interviews were also asked to compare the above equation with $2 s-1=5$. This was in order to see if they were able to conserve equation (Wagner 1977, 1981) under a change of variable. A distinct difference in the type of comment between the two groups shows the superior understanding in this area of those pupils who had used the computer.

The control group featured those who were unsure of the relationship

Pupil C1: $s$ could be 3 as well

Pupil C4 : They could both equal 4

and those who needed to solve both equations

Pupil C2 : Well what I have put is $2 p$ equals 6 and $2 s$ equals 6 ,

Pupil C5 : 2s...add the 1 and 5, 6 er 2 and 2, 6, 3 times, so $s$ is 3 as well.

The experimental (computer) group on the other hand were remarkably consistent in seeing the second equation as being equivalent to the first:

Pupil E1 : I can say that $p$ and $s$ have the same value...its the same sum.

Pupil E5 : The same. Just using a different letter.

All the experimental group responded in this way. These responses show evidence of a global/holistic view of the equations which has enabled the students to develop the understanding of conservation of equation by seeing the common structure of the equations. This concept of conservation of equation under a change of variable was further tested with several of the children by using an extension to the first question :

Solve $2(p+1)-1=5$.

The insight of the computer group pupils is shown by :

Pupil E1 : Yes, $p$ equals 2.

Interviewer : How did you work that out then?

Pupil E1 : Well its the same, but its plus 1, so minus 1 add 3.

Pupil E2 : Oh it would be 2.

Interviewer : Can you tell me why?

Pupil E2 : Because $p$ plus 1 if that's 3 its the same as the last one only the $p$ is less because you've got to add 1 to the sum. 
Deep and powerful insights such as these, facilitated by a global/holistic view leading to the structure of the equations, was not matched by the controls. Instead we have :

Pupil C6 : Say $p$ plus 1, there is already 1 plus $p$ plus another one, I'd say that was $2 p$, and then outside plus another 2 that is 4 minus 1 is 3 , I would say.

Interviewer: So what is the answer?

Pupil C6 : $p$ equals 1 , I would say.

Many would agree that the type of algebraic equation where there are variables on both sides of the equation is conceptually considerably more difficult, since they involve one in algebraic manipulation (i.e. of variables) rather than arithmetic (e.g. Herscovics and Kieran 1980). Although the work done by neither group had involved them in being taught how to solve this type of equation, several of the interviewees were given one to solve. One of the features of relational understanding one might expect to see is that it would be more easily extensible than instrumental understanding, more readily applicable to new areas of mathematics. The ways in which the two groups tackled the question :

Solve $3 x-5=2 x+1$

again seemed to indicate a superior understanding on the part of those who had used the computer.

For instance, one control pupil began to manipulate the expression instrumentally:

Pupil C6 : I'd say it was minus $2 x$ and here you've got $3 x, 2 x$ plus $1 x$, so I'd put that as $1 x$.

[Writes $3 x-5=2 x+1=1 x$ ]

Interviewer : And is that the answer?

Pupil C6 : Yes.

Here the pupil clearly realized that progress might be made by subtracting $2 x$ but carried it out in a mechanical way which led to an incorrect result on which he did not wish to elaborate.

Another control pupil lost sight of the objective all together :

Pupil C3 : I'm trying to work out how you could take 5 from that to leave that.

Interviewer : Can you see any way of doing it?

Pupil C3 : You would have to find the value of $x$ before you could start.

In contrast, the experimental pupils responded more purposefully:

Pupil E1 : Well the value of $x$ must be the same because it's in the same sum... I'm thinking that maybe take $x$ times some number away from both sides. That wouldn't leave anything in there to go on. You'd have nothing there if you take $2 x$ away and $1 x$ minus 5 equals plus 1 .

[Writes $x-5=+1$ ] 
Interviewer : So how might you do it now?

Pupil E1 : I was thinking maybe get rid of this and forget about that 4 by putting, adding 5 to both sides - that should do it - so it would be $3 x$ equals $2 x$ plus $6 \ldots$ try to take $x$ away.

[Writes $3 x=2 x+6$ ]

Shortly after this he solved the equation.

Another experimental pupil started with what seemed like a serialist/analytic approach, but accompanied his actions with clear reasons for the steps he was taking, suddenly making a mental connection that enabled him to solve the problem.

Pupil E2 : You would add 5 to that to get rid of the minus 5 and then that plus 6 so it would be $3 x$ equals $2 x$ plus $6 \ldots .$. Well that plus 6 has got a bigger $x$ because $2 x$ plus 6 equals $3 x$, that means another 6 would be equal to $x$, so make that $3 x$ as well...Well $x$ equals 6 .

In the middle of the question the pupil is versatile enough to change viewpoint (possibly as a result of a cognitive conflict) to a global/holistic one and see the equation in terms of its balancing structure, equating the extra $x$ with 6 .

\section{The Questionnaire}

A second means of testing the pupils' understanding which was employed was a questionnaire. This probed their understanding and view of algebraic notation and although it did not allow the opportunity to follow up answers in the way that an interview does, it had the advantage of wider coverage, being given to 147 pupils.

The questionnaire included three types of questions:

- to explain, with reasons, whether two algebraic expressions were equal or not,

- to explain to an imaginary visitor from Mars the meaning of some algebraic notation,

- harder algebraic questions, beyond the level studied.

\begin{tabular}{||l|c|c|c|c||}
\hline \multicolumn{1}{|c|}{ Question } & $\begin{array}{c}\text { Experimental } \\
\text { proportion } \\
\text { correct }\end{array}$ & $\begin{array}{c}\text { Control } \\
\text { proportion } \\
\text { correct }\end{array}$ & $z$ & $p$ \\
\hline Is $\frac{6}{7}$ the same as $6 \div 7 ?$ & 0.76 & 0.44 & 3.38 & $<0.0005$ \\
\hline Is $2+3 c$ the same as $5 c ?$ & 0.41 & 0.31 & 1.33 & n.s \\
\hline Is 2 $a+b)$ the same as $2 a+2 b ?$ & 0.57 & 0.31 & 2.69 & $<0.0005$ \\
\hline Solve $13-y=2 y+7$ & 0.43 & 0.27 & 1.83 & $<0.05$ \\
\hline Simplify $5 h-(3 g+2 h)$ & 0.24 & 0.08 & 2.16 & $<0.025$ \\
\hline Solve $17-3 e>2$ & 0.31 & 0.13 & 2.37 & $<0.01$ \\
\hline
\end{tabular}

Table V : A Comparison of Some Questionnaire Facilities 


\begin{tabular}{||l|c|c|c|c||}
\hline Question & $\begin{array}{c}\text { Experimental } \\
\text { proportion } \\
\text { making error }\end{array}$ & $\begin{array}{c}\text { Control } \\
\text { proportion } \\
\text { making error }\end{array}$ & $z$ & $p$ \\
\hline $3+m=3 m$ & 0.08 & 0.27 & 2.54 & $<0.01$ \\
\hline$a b=a+b$ & 0.06 & 0.23 & 1.77 & $<0.05$ \\
\hline$b-2 \times c=(b-2) c$ & 0.09 & 0.23 & 1.71 & $<0.05$ \\
\hline $3+2 m=5 m$ & 0.04 & 0.13 & 1.57 & n.s \\
\hline
\end{tabular}

Table VI : A Comparison of Some Specific Errors

Table $\mathrm{V}$ shows the proportions of the pupils giving correct answers to selected questions and table VI gives the proportion making a specific error. (In both tables there were 78 experimental and 69 control pupils, with no significant difference between pre-test means.) These results, and the fact that the controls did not perform significantly better than the experimental group on any question, appear to confirm the superior understanding of algebraic notation of the computer group. Moreover, the experimental students seem to have a better, more global, view of the notation which in turn has reduced the occurence of some of the more common notational errors such as conjoining in addition and the wrong use of brackets.

Of the three questions cited in table $\mathrm{V}$ asking if two expressions are equivalent, the experimental students perform worse than than the control students in every case. The least well answered of these is the (non)equivalence of $2+3 c$ and $5 c$, illustrating the persistence of the parsing obstacle and the probable reading of the symbolism from left to right. The experimental students perform better, but not at a statistically significant level.

The first of these three questions is a significant instance of the processproduct obstacle in which many of the controls did not consider the two notations as the equivalent because

$$
\text { " } \frac{6}{7} \text { is a fraction, } 6 \div 7 \text { is a sum". }
$$

This reveals the perception of $6 \div 7$ as a process involving value-operationvalue rather than as a global entity - the single number - produced by this process. This was not such a problem for the computer group.

The other questions reveal the understanding of the computer group to be superior in its extensibility to the more difficult questions with their requirement of deeper understanding.

\section{Reflections}

The experiments show that the students using the Dynamic Algebra Module are more versatile in their thinking than the students following a traditional course. They are better able to cope with the parsing obstacle, being significantly better at interpreting symbolism and having fewer parsing errors such as $2+3 a=5 a$ or $3+m=3 m$. 
They are better able to cope with the process-product obstacle, evidenced by the fact that they are more likely to consider equivalent expressions such as $2(a+b)$ and $2 a+2 b$ to be the same. They are better at conserving equations and are less likely to feel the need to go through a solution process to solve an equation equivalent to one which they have just solved. They are more likely to be able to chunk information, for instance in seeing that $p+1$ can be treated as the variable in the equation $2(p+1)-1=5$, whilst the control pupils are more likely to feel the need to process the information by multiplying out, collecting terms, and solving for $p$.

They are more likely to be able to think of expressions as objects, thus overcoming the lack of closure obstacle and seem to have no problem with the expected answer obstacle.

Those taking the Dynamic Algebra Module show a reversal of the accepted order of difficulty of certain concepts: they are initially better at certain concepts considered more difficult in the CSMS tests (such as solving the inequality $a+3>7$ ) and less good at routine algebraic skills (such as simplifying $3 b-b+a)$. However, after a brief revision of routine skills, they improved to match the control students at routine problems whilst retaining their superiority in higher order skills.

This is consistent with Heid's work in calculus (Heid 1988) where it was shown that students who use the computer for conceptual work and only carry out routine manipulation for a short time at the end of the course were better at higher level conceptual problems than control students following a traditional course and not significantly different in ability with routine manipulation.

There is mixed evidence over the very long term improvements caused by a short three-week period of study using the computer different in nature from the rest of the work of the students. The initial pilot study, in which the experimental students used a versatile computer approach to algebra and the control students did no algebra, showed a maintained difference in performance over a year later even after further study of algebra by both groups. The main study revealed the superior medium term performance of the experimental students following a versatile computer scheme over the control students following a traditional scheme, but failed to reveal a statistically significant difference eighteen months later during which time there was no further computer work.

There is evidence that the relative degree of difficulty of concepts may differ in a computer paradigm from a traditional paradigm, suggesting the need for further research and the reassessment of fondly held beliefs of what is hard and what is easy. 
Finally, although the Dynamic Algebra Module only tackles the initial phases of letter as a label for a number or set of numbers, and equivalence of expressions through evaluation, there is evidence that the treatment has an immediate extension to linear equations.

\section{References}

Bogen J.E. 1969: "The Other Side of the Brain: 2. An Appositional Mind", Bulletin of the Los-Angeles Neurological Society 34, 1969, pp. 135-162.

Brumby M.N. 1982: "Consistent differences in cognitive styles shown for qualitative biological problem-solving", British Journal of Educational Psychology, 52, 244-257.

Descartes R. 1628: Regulae ad Directionem Ingenii, (Published posthumously and also translated into English as "Rules for the Direction of the Mind", in The Philosophical Works of Descartes, E.S. Haldane and G.R.T. Ross, 1934).

Gardiner A. 1988: "WIII computers really count?", Micromath, 4 1, 31-32.

Gazzaniga M.S. 1974: "Cerebral Dominance Viewed as a Decision System”, in S.J. Dimond and J.G. Beaumont (Eds.), Hemisphere Function in the Human Brain, Elek Science, London, pp. 367382.

Gazzaniga M.S. 1985: The Social Brain, Basic Books, New York.

Heid M.K . 1988: "Resequencing Skills and Concepts in Applied Calculus using the Computer as a Tool", Journal for Research in Mathematics Education, 19 1, 3-25.

Herscovics N. and Kieran C. 1980: "Constructing Meaning for the Concept of Equation", Mathematics Teacher 73, 572-580.

Keiran C. 1981: "Pre-algebraic notions among 12 and 13 year olds", Proceedings of PME 5, Grenoble, 158-164.

Krutetskii V. A.: 1976, The Psychology of Mathematical Abilities in Schoolchildren, (translated from the Russian by J. Teller), Chicago.

Küchemann D.E. 1981: “Algebra”, in Children's Understanding of Mathematics : 11-16, K.M. Hart (Ed), John Murray, pp. 102-119.

Leitzel J.R. \& Demana F. 1988: "Establishing fundamental concepts in algebra through numerical problem-solving", NCTM 1988 Year Book.

Matz M. 1980: "Towards a Computational Theory of Algebraic Competence", Journal of Mathematical Behaviour, 3(1) 93-166.

Poincaré H. : 1903, La Science et l'hypothèse, included in The Foundations of Science (translated by Halsted G.B., 1913) The Science Press.

Popper K.R. and Eccles J.C. 1977: The Self and Its Brain, Berlin, Springer.

Rosnick P. and Clement J. 1980: "Learning Without Understanding : The Effect of Tutorial Strategies on Algebra Misconceptions", Journal of Mathematical Behaviour, 3(1), 3-27. 
Seamon J.G. 1974: "Coding and Retrieval Processes and the Hemispheres of the Brain", in S.J.

Dimond and J.G. Beaumont (Eds.), Hemisphere Function in the Human Brain, Elek Science, London, pp.184-203.

Sperry R.W., Gazzaniga M.S. and Bogen J.E. 1969: "Interhemispheric Relationships: The Neocortical Commissures: Syndromes of Hemispheric Deconnection", in P.J. Vinken and G.W. Bruyn (Eds.), Handbook of Clinical Neurology, Vol. 4, North Holland Publishing Co., Amsterdam.

Skemp R.R. 1976: "Relational understanding and instrumental understanding", Mathematics Teaching 77, 20-26.

Skemp R.R., 1979: Intelligence, Learning and Action, Wiley.

Tall D.O. 1986: Building and Testing a Cognitive Approach to the Calculus Using Interactive Computer Graphics, Ph.D. thesis, University of Warwick.

Tall D.O. 1990: "Concept Images, Generic Organizers, Computers and Curriculum Change", For the Learning of Mathematics, 9, 3, 37-42.

Thomas M.O.J. 1985: The Effects of BASIC Computer Programming on the Understanding of the Use of Letters as Variables in Algebra, unpublished M.Sc. thesis, University of Warwick.

Thomas M.O.J. 1988: A Conceptual Approach to the Early Learning of Algebra Using a Computer, unpublished Ph.D. thesis, University of Warwick

Thomas M.O.J. and Tall D.O. 1986: "The Value of the Computer in Learning Algebra Concepts.", Proceedings of the 10th Conference of PME, London, 313-318 .

Thomas M.O.J. and Tall D.O. 1988: "Longer-Term Conceptual Benefits From Using A Computer in Algebra Teaching", Proceedings of the 12th Conference of PME, Veszprem, Hungary, 601608.

Thomas M.O.J. and Tall D.O. 1989a: "Dynamic Algebra", in Secondary Mathematics with Micros, Mathematical Association.

Thomas M.O.J. and Tall D.O. 1989b: “"Verbal Evidence for Versatile Understanding of Variables in a Computer Environment", Proceedings of P.M.E., Paris, volume 3, 213-220.

Wagner S. 1977: Conservation of Equation, Conservation of Function and Their Relationship to Formal Operational Thinking, unpublished doctoral dissertation, New York University.

Wagner S. 1981: "Conservation of equation and function under transformations of variable", Journal for Research in Mathematics Education, 12, 197-118.

Wagner S., Rachlin S.L. and Jensen R.J. 1984: Algebra Learning Project - Final Report, Department of Mathematics Education, University of Georgia. 